\title{
Atenção integral no cuidado familiar do idoso: desafios para a enfermagem gerontológica no contexto da estratégia de saúde da família
}

Comprehensive health care in family care for the elderly: challenges for gerontological nursing

in the context of the family health strategy

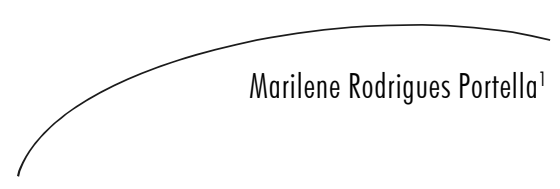

Resumo

O tema cuidador do idoso tem sido objeto de debates nos encontros científicos da enfermagem brasileira, nos eventos da gerontologia nacional e internacional. Este artigo reflete a palestra apresentada na mesa redonda "A Multidimensionalidade do Processo de Envelhecimento, a Enfermagem e o Cuidado Familiar", na VIII Jornada Brasileira de Enfermagem Geriátrica e Gerontológica realizada na Universidade do Estado do Rio de Janeiro, no mês de abril de 2010. Aborda, em especial, o papel da enfermagem, na atenção básica, junto ao cuidador familiar considerando os contextos urbano e rural.

\section{Abstract}

The theme of caregivers of elderly people has been the subject of debate in scientific meetings of Brazilian nursing, at gerontology events. This article reflects the speech given at the roundtable "The multidimensionality at the Aging Process, Nursing and the Family care," in the VIII Brazilian Conference of Gerontological and Geriatric Nursing held at the Universidade do Rio de Janeiro, in April 2010. It addresses in particular the role of nursing in primary care with the family caregiver considering the urban and rural context.

\footnotetext{
Correspondência / Correspondence

${ }^{1}$ Marilene Rodrigues Portella

Universidade de Passo Fundo. Instituto de Ciências Biológicas, Enfermagem.

Rua Teixeira Soares 780 - Centro

99010-080 - Passo Fundo, RS, Brasil

E-mail: portella@upf.br
}

Este artigo foi elaborado a partir de palestra proferida na VIII Jornada Brasileira de Enfermagem Geriátrica e Gerontológica realizada na Universidade do Estado do Rio de Janeiro, em abril de 2010.

\section{Palavras-chave:}

Enfermagem Geriátrica.

Gerontologia. Cuidadores.

Atenção Básica.

Key words: Geriatric Nursing. Geriatrics. Caregivers. Primary Health Care 


\section{INTRODUÇÃO}

Temos que compreender que a temática central desta mesa-redonda, "Multidimensionalidade do Processo de Envelhecimento, a Enfermagem e o Cuidado Familiar" traduz o anseio da enfermagem gerontológica brasileira em discutir questões que envolvem políticas públicas de atenção e participação sistemática dos profissionais de saúde no cuidado domiciliar da pessoa idosa. Neste contexto, a enfermagem, a família, o idoso e seu cuidador são personagens de uma mesma história. Como atores do cotidiano, no palco da vida, mostram cenários diversos; cenas de momentos de vida específicos, nas quais se podem vislumbrar valores, metas e estruturas diferenciadas. Um encontro de diferentes saberes e práticas, por vezes, nem sempre bem compreendido de ambas as partes.

\section{ENFERMAGEM E CUIDADO FAMILIAR DO IDOSO}

O envelhecimento populacional é uma realidade que chama atenção pela grande heterogeneidade do grupo etário idoso, seja pelas condições sociais, política, econômica e principalmente, de saúde. Doenças crônicas e múltiplas tendem a se manifestar com muita frequência no grupo etário idoso, causando limitações importantes e requerendo cuidados prolongados: assim, vale lembrar que, no Brasil, cerca de $40 \%$ do tempo vivido pelos idosos é sem saúde ${ }^{1}$.

Desta forma, pensar sobre os grandes desafios $\mathrm{da}$ enfermagem frente ao cuidado familiar do idoso requer a compreensão de que, com a implementação da estratégia de Saúde da Família (ESF) na Atenção Básica (AB), os cuidados domiciliários de saúde tornaram-se parte integrante das ações em saúde nesse nível de atenção ${ }^{2}$. Embora o cuidado domiciliar à saúde seja uma prática que remonta à própria existência das famílias como unidade de organização social, devemos atentar para inúmeras situações, em que, sob o discurso da transformação do modelo tecnoassistencial por meio da implementação maciça da saúde da família, ocorrem equívocos, cujo resultado se expressa na transferência dos cuidados hospitalares para os cuidados prestados na comunidade ${ }^{3}$.

Inúmeras situações de dependência cronicamente assumidas pelas famílias revelam que pessoas com doença avançada e progressiva são cuidadas por cuidadores informais, sejam eles familiares ou não4. Estes, por outro lado, desempenham tarefas que lhes são atribuídas, na maioria das vezes, sem orientação adequada, com suporte ineficaz por parte do sistema de saúde. Tal condição, somada a alteração da rotina e tempo dispensado no cuidado, tem impactos sobre a qualidade de vida do cuidador. Quanto maior a duração do cuidado, pior a qualidade de vida do cuidador ${ }^{5-7}$.

Pensar ações em saúde no contexto gerontológico no nível da $\mathrm{AB}$ requer o reconhecimento de que o cuidador não apenas tem utilidade e importância no setor saúde, como aliado da equipe no processo de cuidado, mas também é um usuário carente de zelo. Assim, o papel da Enfermagem Gerontológica, na atenção integral ao idoso, passa pela identificação das necessidades do cuidador familiar.

\section{NECESSIDADES DOS CUIDADORES}

Quando se pensa na atenção integral ao cuidador familiar do idoso, é imprescindível o diagnóstico das necessidades dos cuidadores, o que requer levar em conta que, na identificação das mesmas, há algumas dificuldades a serem superadas, quais sejam:

\section{- Reconhecimento das necessidades veladas}

Muitos cuidadores demonstram certa relutância em comunicar as suas necessidades aos profissionais, por pensarem não ser aceitável fazêlo. Em alguns, podemos observar o constrangimento, pois diante do sofrimento alheio não querem colocar suas necessidades à frente das necessidades da pessoa cuidada. Por outro lado, há aqueles que se sentem receosos, pois tais colocações poderão ser objeto de julgamento, e sua condição de cuidador pode ser interpretada de modo inadequado. Outros 
entendem que isto faz parte da vida; é uma situação "sem saída", na qual os desgastes e as preocupações são contingências do acaso, portanto, não podem ser minimizados. Há ainda casos em que as dificuldades ficam veladas pelo fato de os profissionais de saúde não mencionarem questões relevantes acerca do cuidado, como forma de orientação desse cuidador ${ }^{7-9}$.

\section{- Variabilidade das necessidades ao longo do continuum do processo cuidativo}

Em circunstância de cronicidade, o processo cuidativo é prolongado e os cuidadores, ao longo do continuum, enfrentam diferentes momentos de acordo com o curso da evolução da enfermidade da pessoa cuidada, vivenciando situações em que ora estão cuidando no domicilio, ora acompanhando o idoso nos períodos de hospitalização. Acompanhar/ cuidar no hospital difere muito no que diz respeito ao cuidado no domicílio frente às condições estruturais.

Nas instituições hospitalares, mesmo que seja um transtorno para o cuidador coordenar o andamento doméstico e familiar concomitante ao cuidado, ele tem a vantagem de contar com a estrutura dos serviços de saúde; por outro lado, no domicílio, são frequentes os casos em que há indisponibilidade de suporte de apoio, da parte dos serviços de saúde, da escassez de recursos que advém com o tratamento prolongado, ou até mesmo da insuficiência ou ausência de familiares ${ }^{7-}$ 11.

\section{PRINCIPAIS NECESSIDADES DOS CUIDADORES}

Nos estudos que temos encaminhado encontramos pontos em comum comparados aos de nossos pares. $\mathrm{Na}$ abordagem da questão, as necessidades podem ser assim classificadas: relacionadas à tarefa cuidativa e relacionadas ao cuidado de si.

\section{- Relacionadas à tarefa cuidativa}

Cuidar de um familiar idoso, mesmo que seja ação entendida como extensão das atividades domésticas, requer da parte dos cuidadores um planejamento, visto que a rotina, aos poucos ou bruscamente, é alterada. O grande desafio neste momento é estar preparado para tal. Os cuidadores necessitam de treinamento voltado paraa realidade de cada caso; carecem de noções de gestão/administração do cuidado, pois, na maioria das vezes, nem sequer recebeminformações elementares sobre os cuidados ao doente e orientação sobre o uso da medicação, quiçá contar com o apoio dos profissionais de saúde. ${ }^{7,11-13}$

A tarefa de cuidar é vista como uma continuidade da tradição familiar ${ }^{7,8}$, como retribuição e gratidão; para alguns pode ser motivos de satisfação, para outros obrigação ${ }^{8,11-13}$; e quando há disfuncionalidade familiar os cuidadores necessitam de apoio na gestão da comunicação e funcionamento familiar ${ }^{2,14}$.

\section{- Relacionadas ao cuidado de si}

O tempo absorvido com o cuidado do idoso provoca redução do lazer, do convívio com a família e os amigos, afeta a vida particular dos cuidadores ${ }^{11,13,15}$, assim uma necessidade imprescindível é o reconhecimento do abandono de si em razão da sobrecarga de atividade. Estudos confirmam que a exaustão e a doença se tornam uma incidência inevitável na vida dos cuidadores ${ }^{13,15-17}$.

Ter consciência dos seus limites é imperioso para os cuidadores; reconhecer o momento de pedir ajuda é cogente na relação cuidativa, todavia a interpretação de tal necessidade, na maioria das vezes, não acontece de modo espontâneo, não é algo fácil para os mesmos ${ }^{13,17}$. Coerente com o cuidado de si, trabalhar a sobrecarga emocional trata-se de uma necessidade elencada nos estudos sobre os cuidadores de pessoas portadoras de sofrimento psíquico e, na atenção básica, no que diz respeito aos cuidadores de idosos, estamos falando de uma ocorrência comum ${ }^{13,18}$. 


\section{A ENFERMAGEM GERONTOLÓGICA NA ATENÇÃO BÁSICA EM SAÚDE}

O fortalecimento da atenção básica tem como finalidade principal a consolidação e qualificação da ESF como modelo de atenção básica à saúde e como centro ordenador das redes de atenção à saúde do SUS, o que exige profissionais aptos a desenvolver atitudes efetivas e de impacto na atenção à saúde do idoso ${ }^{19}$. Tais atitudes pressupõem o cuidado integral e humanizado, mas encontramos realidades em que $o$ constrangimento avassala as pessoas idosas e/ou seus cuidadores, devido à cobrança (implícita ou explícita) quanto aos cuidados, não só de membros da família ou da comunidade, como também dos próprios profissionais de saúde ${ }^{20}$.

A ESF tem sido de fundamental importância na promoção da saúde familiar, em especial dos idosos, porque auxilia no controle de uma série de enfermidades, e também contribui substancialmente na melhoria da qualidade de vida daqueles com quem interage de forma efetiva. Apesar dos avanços no que se refere aos princípios norteadores do SUS e às estratégias propostas, percebemos que ainda se enfrenta uma série de dificuldades, destacando-se a fragmentação do processo de trabalho e das relações entre os diferentes profissionais; falta de complementaridade entre rede básica e o sistema de referência; precária interação nas equipes e despreparo para lidar com a dimensão subjetiva nas práticas de atenção.

Trabalhando na docência, acompanhando aluno em campo de prática na rede de atenção básica e convivendo diariamente com a rotina dos profissionais da ESF e também idosos, familiares e seus cuidadores, sinto-me impelida a comentar que muitos profissionais estão aptos ao desempenho de procedimentos tecnicistas, porém despreparados para a escuta sensível. Seriam as "lacunas" na formação as responsáveis por tal desempenho? Tal conduta poderia ser atribuída à banalização do sofrimento alheio por parte dos profissionais de saúde? Neste momento não cabe tal discussão, nem é o nosso propósito, mas são questões meritórias de atenção.
Temos que considerar também que a ESF se operacionaliza a partir de uma Unidade Básica de Saúde cuja localização é o espaço urbano. $\mathrm{O}$ contexto rural conta apenas com suporte dos Agentes Comunitários de Saúde (ACS) nas visitas domiciliares regulares, que percorrem grandes distâncias geográficas na microárea de atuação e, muitas vezes, estão despreparados para identificar as situações de risco. Essa realidade revela maior demanda de tempo - exigência além da capacitação em função das amplas solicitações demandadas pelo usuário e do confronto com as misérias humanas cuja solução excede sua compreensão e atuação ${ }^{21}$. A convivência com os mais variados problemas da população gera desgaste do ACS, por vezes, incorrendo no seu adoecimento ${ }^{22}$.

No que diz respeito ao atendimento da equipe de saúde no meio rural, observamos ações pontuais nos grupos de hipertensos e diabéticos. As reuniões se caracterizam pela realização de palestras e distribuição de medicamentos, o que obriga o usuário comparecer - ou seja, segue o modelo que prioriza a lógica da medicalização ${ }^{23}$.

DESAFIOS PARA A ENFERMAGEM GERONTOLÓGICA EM RELAÇÃO À ATENÇÃO INTEGRAL AO CUIDADOR FAMILIAR

Discutir os desafios para a Enfermagem em relação à atenção integral à saúde do idoso, tema central dessa jornada, concebendo a multidimensionalidade do processo de envelhecimento, a enfermagem e o cuidado familiar, assunto específico desta mesa-redonda, reveste-se de grande veemência, tendo em conta a complexidade, sobretudo quando a assistência gerontológica é direcionada por um conceito ampliado do processo saúde/doença, com vistas à melhoria da qualidade de vida dos gerontes.

Atuar desta forma é o desafio que se coloca também aos serviços de saúde ${ }^{22}$. Seguindo essa assertiva, ressaltamos que nossa participação neste fórum de discussão, a Jornada Brasileira de Enfermagem Geriátrica e Gerontológica, caracteriza a enfermagem gerontológica como protagonista da mudança de paradigma, pois 
estamos discutindo o papel da Enfermagem frente à "saga" do ser cuidador de idoso e a família cuidadora. Assim, nossa proposta consiste em refletir sobre a condição do cuidador familiar e agir, no intuito de propor a implementação de propostas educativas/cuidativas observando os "muitos brasis" que aqui se apresentam enquanto cenários de prática e de centros de formação.

Como parte deste desafio, destaca-se a perspectiva de trabalho do Departamento Científico de Enfermagem GerontológicaDEGER-ABEn, expressa no Regimento Interno, aprovado em 7 de fevereiro de 2010, sobretudo no Art. $4^{\circ}$, inciso III - Realizar, junto às seções, Regionais e Núcleos da ABEn, cursos de aperfeiçoamento e treinamentos para Enfermeiros e Técnicos de Enfermagem, que favoreçam a capacitação para o cuidado de pessoas idosas, complementando no inciso IV - propor medidas

\section{REFERÊNCIAS}

1. Camarano AA, Kanso S, Mello JL. Como vive o idoso brasileiro? In: Camarano AA (Org.). Os novos idosos brasileiros. Muito além dos 60 ? Rio de Janeiro: IPEA, 2004:25-59.

2. Brasil. Ministério da Saúde. Secretaria da Atenção a Saúde. Departamento de Atenção Básica. Envelhecimento e saúde da pessoa idosa. Brasília: Ministério da Saúde; 2006. Série A. Normas e Manuais Técnicos, Cadernos de Atenção Básica, n.19.

3. Veríssimo C, Moreira I. Os cuidadores familiares informais: cuidar do idoso dependente em domicilio. Pensar em enfermagem 2004; 8(1).

4. Karsch UM. Idosos dependentes: famílias e cuidadores. Cadernos de Saúde Pública 2003; 19(3):861-6.

5. Amendola F, Oliveira MAC, Alvarenga MRM. Qualidade de vida dos cuidadores de pacientes dependentes no programa de saúde da família. Texto contexto - enferm 2008; 17(2): 266-272.

6. Watanabe HAW, Derntl AM. Cuidadores de idosos: uma experiência em Unidade Básica de Saúde - Projeto Capacidade. Mundo Saúde 2005; 29 (4):639-44.

7. Portella MR, Pasqualotti A, Fortes VLF, Scortegagna HM, Strehl JM, Dalbosco J. necessárias à defesa e consolidação do trabalho em Enfermagem Gerontológica.

A relevância do DEGER-ABEn insere-se na busca de uma compreensão da realidade vivida pelos profissionais da enfermagem na atenção integral ao idoso, incluindo os atuantes nas ESFs e na proposição de estratégias de educação permanente no intuito de caminhar na direcionalidade da construção e consolidação de um novo modelo. Segundo Marin et al. ${ }^{24}$, tratase do vislumbre de mudança no modelo de atenção.

A partir daí, considera-se lançado o desafio para que, daqui a dois anos, no próximo encontro, na XIX Jornada Brasileira de Enfermagem Geriátrica e Gerontológica possamos estar apreciando os relatos dos primeiros feitos deste departamento.

Cuidar de um familiar idoso: repercussões no curso da vida. In: Portella MR, Pasqualotti A, Gaglietti M. (Org.). Envelhecimento humano: saberes e fazeres. Passo Fundo/RS: UPF Editora, 2006:19-31.

8. Silveira TM, Caldas CP, Carneiro TF. Cuidando de idosos altamente dependentes na comunidade: um estudo sobre cuidadores familiares principais. Cad. Saúde Pública 2006; $22(8): 1629-38$.

9. Orrell M, Cooper C, Braithwaite S. Do carers' needs assessments make a difference? Results from the Forget me not study. Age and Ageing 2006; 35(4):444-5.

10. Celich KL, Batistella M. Ser cuidador do familiar portador de Doença de Alzheimer: vivências e sentimentos desvelados. Cogitare Enferm 2007; 12(2): 143-49.

11. Cattani RB, Girardon-Perlini NMO. Cuidar do idoso doente no domicílio na voz de cuidadores familiares. Rev. Eletrônica. Enferm 2004; 06(02): 254271.

12. Bocchi SCM. Vivenciando a sobrecarga ao vir-aser um cuidador familiar de uma pessoa com acidente vascular cerebral (AVC): uma análise do conhecimento. Rev Latino-am Enfermagem 2004; 12(1):115-21. 
13. Agich GJ. Dependência e autonomia na velhice: um modelo ético para o cuidado de longo prazo. São Paulo: Loyola, Centro Universitário São Camilo; 2008.

14. Kristjanson LJ. Family Care. In: Textbook of Palliative Medicine. Hodden Arnold; 2006.

15. Sommerhalder C, Neri AL. Avaliação subjetiva da tarefa de cuidar: ônus e benefícios percebidos por cuidadoras familiares de idosos de alta dependência. In: Neri AL. (org). Cuidar de idosos no contexto da família: questões psicológicas sociais. Campinas: Átomo Alínea; 2002.

16. Gonçalves LHT, Alvarez AM, Santos SMA. Cuidadores leigos de pessoas idosas. In: Duarte YAO, Diogo MJD (org). Atendimento domiciliar: um enfoque gerontológico. São Paulo: Atheneu; 2000.

17. Laham CF. Percepção de perdas e ganhos subjetivos entre cuidadores de pacientes atendidos em um programa de assistência domiciliar. [Dissertação]. São Paulo. Faculdade de Medicina do Estado de São Paulo; 2003.

18. Borba LO, Schwartz E, Kantorski LP. A sobrecarga da família que convive com a realidade do transtorno mental. Acta paul. enferm. [periódico na Internet]. 2008 [acesso em 2010 Jun 10]; 21(4):588-594. Disponível em: http:/ /www.scielo.br/ scielo.php?script $=$ sci arttext\&pid $=$ S010321002008000400009\&lng=pt. doi: 10.1590/S010321002008000400009.
19. Silva AA, Borges MMMC. Humanização da assistência de enfermagem ao idoso em uma unidade de saúde da família. Revista Enfermagem Integrada. 2008; 1(1): [Acesso em 2010 maio.02]. Disponível em: URL: http:// www.unilestemg.br/enfermagemintegrada/ artigo/v1/andreia_silva_e_marta_borges.pdf

20. Brêtas ACP, Yoshitome AY. Conversando com quem gosta de cuidar do idoso no domicilio. In: Duarte YAO, Diogo MJD (org). Atendimento domiciliar: um enfoque gerontológico. São Paulo: Atheneu; 2000.

21. Portella MR. A pessoa idosa no contexto rural da $6^{\circ}$ CRS [CD-ROM] In: Creutzberg M. (org.). VII Jornada Brasileira de Enfermagem Geriátrica e Gerontológica, 2008. Porto Alegre. Anais. Brasília: ABEn; 2008.

22. Wai MFP, Carvalho AMP. O trabalho do Agente Comunitário de Saúde: fatores de sobrecarga e estratégias de enfrentamento. Rev. enferm. UERJ. 2009; 17(4): 563-8.

23. Coriolano MWL, Lima LS. Grupos focais com Agentes Comunitários de Saúde: subsídios para entendimento desses atores sociais. Rev. enferm. UERJ. 2010; 18(1): 92-6.

24. Marin MJS, Martins AP, Marques F, Feres BOM, Saraiva AKH, Druzian S. A atenção à saúde do idoso: ações e perspectivas dos profissionais. Rev. Bras. Geriatr. Gerontol. 2008 [Acesso em 2010 jun.02]. Disponível em: URL: http:// www.unati.uerj.br/tse/ scielo.php?script $=$ sci arttext\&pid $=\mathrm{S} 1809$ 98232008000200009\& $\overline{\ln } \mathrm{g}=\mathrm{pt} \& \mathrm{nrm}=$ iso. 